\title{
The study of dynamic capacity-increase online monitoring device of transmission lines based on the beidou satellite accurate time service technology
}

\author{
Yuehua Huang,Denghao Fu,Yu Zheng, Yu Liu,Chuang Li. \\ Department of Electrical Engineering \& New Energy, China Three Gorges University, Yichang, \\ China
}

Keywords: Transmission lines, dynamic Capacity,Line monitoring;, beidou satellite.

\begin{abstract}
Because the static heat capacity is set up on the low probability of occurrence of adverse conditions,this results in the waste of line transmission capacity.This article describes the technique of dynamic Capacity,beidou satellite accurate timing Technology is added to the dynamic capacity-line monitoring system,SCM technology, sensor technology and communication technology are used to measure the conductor temperature, ambient temperature,, humidity,, wind speed, wind direction, sunshine intensity, the data provides reference data for transmission lines dynamic capacity.
\end{abstract}

\section{Introduction}

China's national economy has entered a new period of rapid growth,the electricity load growth of many provinces and territories is over the development of electric power construction,the shortage of the entire grid line corridors is obvious.State Grid Corporation of China put forward that new grid should be built and the transmission capacity of the existing power grid should be increased by using technological innovation and upgrading to improve the efficiency of the power grid in order to meet the growing national economic development and people's living electricity needs[2].The transmission capacity of the line under certain weather conditions can be improved by analyzing real-time monitoring and verification,so that line load can be dynamically adjusted and the transmission capacity of transmission lines can be maximize.However, the communication in real-time monitoring data is widely used the US-developed GPS (Global Positioning System) Timing Products now,the power companies are related to national energy security and national economy,the wildly using of GPS Timing Products will let our power grid construction face with huge potential safety hazards.The products related with GPS that uesd in the Grid construction need to be gradually given up to meet the needs of information security of the grid construction. Therefore, the choice of beidou satellite technology instead of GPS technology is vital,and it's also the future trend of development.

\section{System Design}

The dynamic capacity transmission line online monitoring device is designed to monitor and store the ambient temperature,, humidity, wind speed, wind direction, sunshine intensity, etc[1] of the high voltage lines,and the parameters can be provided to the expert software to adjust the thermal stability limit of transmission lines,so that the transmission capacity of the existing transmission lines can be developed.The Schematic diagram of the online monitoring system shown in Figure 1.

The data transmission of the line monitoring system includes two aspects:the data transmission between the monitoring devices and the monitoring host; the data transmission between the host and the online monitoring center[2].Because of the high cost by using power line transmission,GPRS wireless communication mode is used to to accomplish the transmission of data.GPRS technology has been relatively mature,Its high resource utilization,wide reception range and high transfer rate can meet the requirements of the system. 


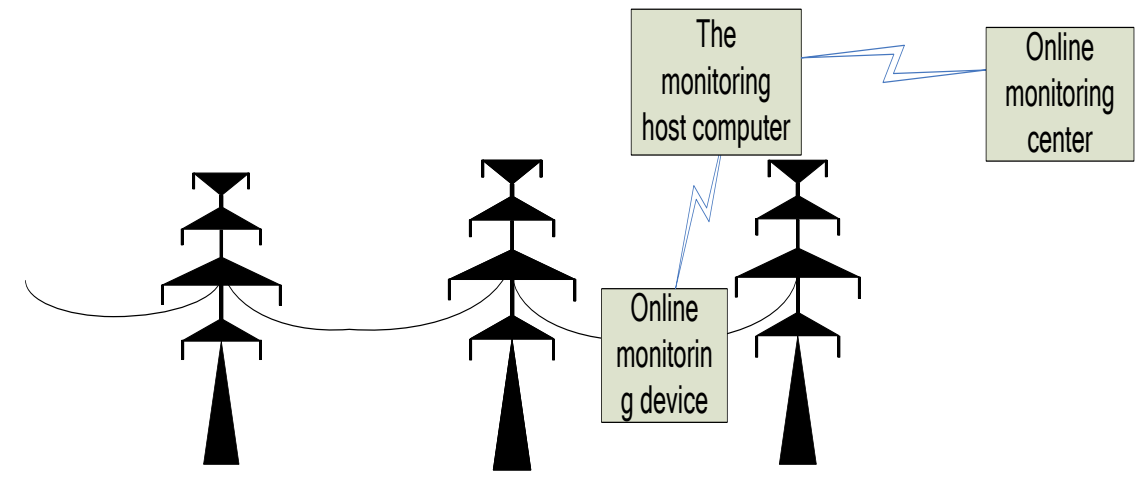

Fig. 1 Schematic diagram of the online monitoring system

\section{The structure of each device}

The diagram of the structure of the system shown in Figure 2.The system includes STM32 MCU control system, GPRS module, Power supply module,, the beidou satellite module, wire temperature sensor, ambient temperature sensor, sunshine intensity sensor, wind speed and direction sensors.

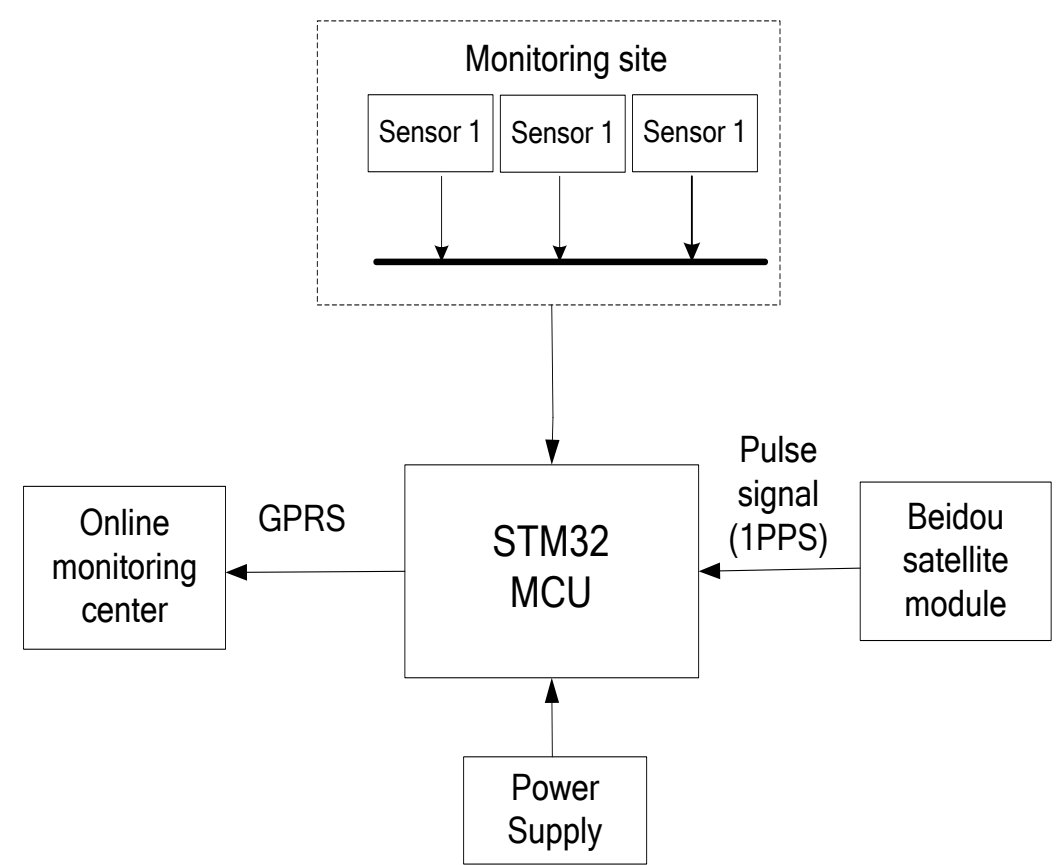

Fig. 2 Schematic diagram of monitoring master station

After passing through the multi-channel analog switch, the signal collected by the sensors is enlarged by the conditioning circuit, then the collected analog value is converted into digital value.Finally, the data is sent to online monitoring center by using the GPRS wireless communication module. The data acquisition and transmission of kinds of sensors require a unified standard time,the clock signal of synchronous acquisition is given by the beidou satellite module. Online monitoring center is equipped with expert analysis software as the upper computer, after being received by the online monitoring center,the data is used to calculate the margin of the line capacity for the staff members referencing.The entire device is powered by the power supply module, because the system needs to run 24 hours a day, so the power is extremely important .Now,there are kinds of ways to take power, such as solar, battery, CT to take power, etc.If solar is used to provide power,it could not supply power reliably in case of rainy days or lack of sunshine,and the battery can supply power stably.So battery and solar is used to supply power to the system. 


\section{The Synchronous acquisition and transmission of the data}

Beidou satellite has two ways of time service:unidirectional time service and bidirectional time service.The schematic of the bidirectional time service is shown in Fig.3 and the schematic of unidirsectional time service is shown in Fig.4.Timing signal is sent from central station at the moment of $t_{0}$,after a delay $t_{1}$, satellite receives the signal,after a delay $t_{2}$, the signal is sent to the user machine,after propagation delay $t_{3}$,satellite receives the signal and after the delay of $t_{4}$, the signal is sent back to the user. So, the Bidirectional propagation delay is $t$ :

$$
t=\left(t_{1}+t_{2}+t_{3}+t_{4}\right)
$$

The system uses a unidirectional timing mode of beidou satellite. The user don't need to interact with the ground central station, but the accurate coordinates of the user should be known, so that the transmission delay of the satellite signal can be calculated and the accurate local time can be obtained. The central control station maintains the accurate time and regularly broadcast timing information, the delay correction value is given to the user. The uplink transmission delay between the central station to satellite, the down link transmission delay between the satellite to the user and other kinds of delay (tropospheric, ionospheric etc) will be produced when transmitting the normative time information.Various of delays are transmitted to the user. The beidou satellite receiver module can correct various of errors, like uplink delay correction, downlink delay correction, best estimate of beidou satellite orbit, Ionospheric delay correction, tropospheric delay correction, earth's rotation effect correction, doppler effect correction and so on. So it can calculate the error of the time and corrects the local time by using the navigational data and some related information, the unidirectional time service timing indicators is $100 \mathrm{~ns}$.

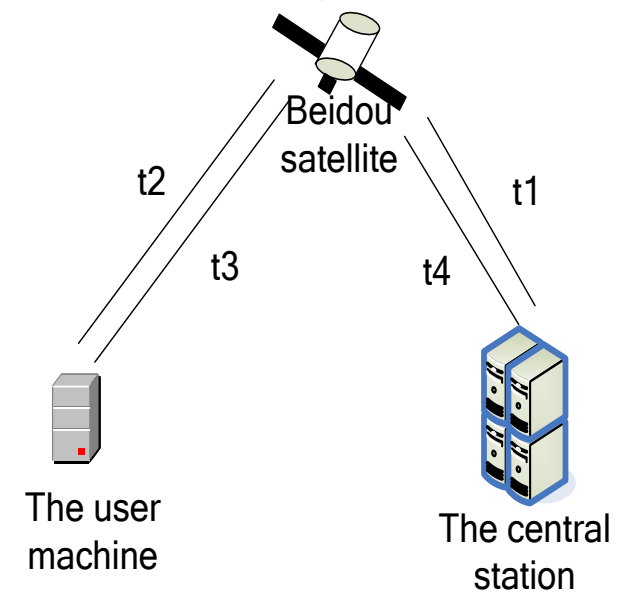

Fig.3 Schematic of unidirsectional time service

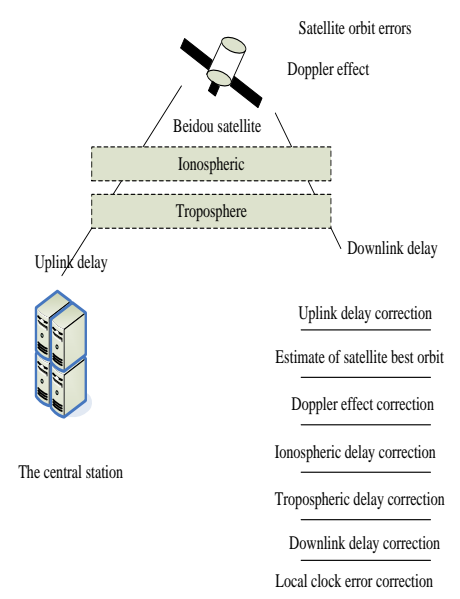

Fig. 4 Schematic of unidirectional time service 
The sensors that are located in the front end of the system, they collect the data that are needed, All kinds of the signals are converted into electrical signals, the conditioning circuit includes amplifying circuit composed by OP747, electrical signal is enlarged to 6 times of the original by the amplifying circuit, then signal is filtered by the low-pass filter composed by OP747,the cutoff frequency of the low-pass filter is $8 \mathrm{KHz}$.Then the filtered signals is sent to the sampling circuit. Signal is sampled by the AD sampling chip ADS1278, the sampling rate is $16 \mathrm{KHz}$. The chip converts the signals from analog format to digital. After serial-to-parallel Converting, data is stored in the storage unit. Beibou Satellites transmits information to the beidou receiver module, then the beidou receiver module decodes the information, so that accurate time information can be obtained. The information provides time standard for synchronous data acquisition. At last the obtained data is wirelessly transmitted to host computer by the GPRS module.

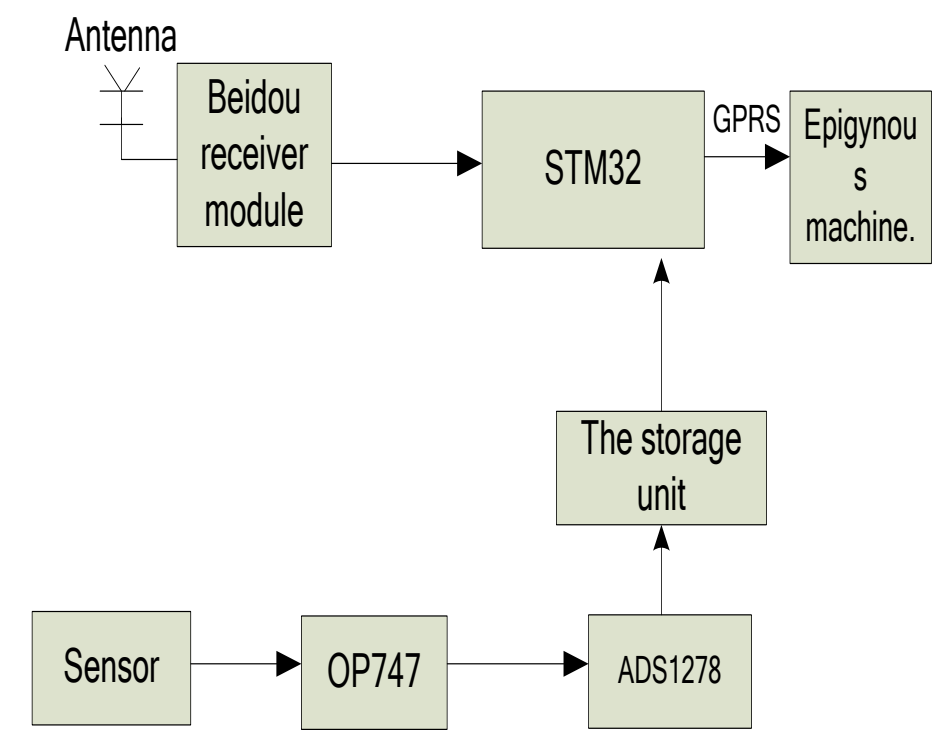

Fig. 5 Schematic diagram of data acquisition and transmission

\section{Anti interference measures of beidou satellite time service}

As the poor working environment and the strong electromagnetic interference around the wire,the 1PPS signal that output from beidou satellite receiver may contain interferences, it may produce fake 1PPS signals,this can lead to improper action of system,so it is necessary to take the corresponding anti-interference measures.The Uniformity between two adjacent pulses can be real-time monitored by using a window signal.If abnormal conditions were founded,1PPS signal will be shielded immediately.There are two methods to produce the window signal,one method is software method and the other one is hardware method.The software method is using the software setting counter to produce a window signal on the rising edge of 1PPS signal on the base of 1PPS interrupt output from the beidou satellite receiver. The hardware method refers to generate a window signal in CPLD or FPGA by using 1PPS and counter.As is shown in fig.6,it uses hardware windowing method,1PPS signal is gated by using "and" gate logic,it is completed by the shielding signal generated by the FPGA/CPLD application system and microcontroller. 


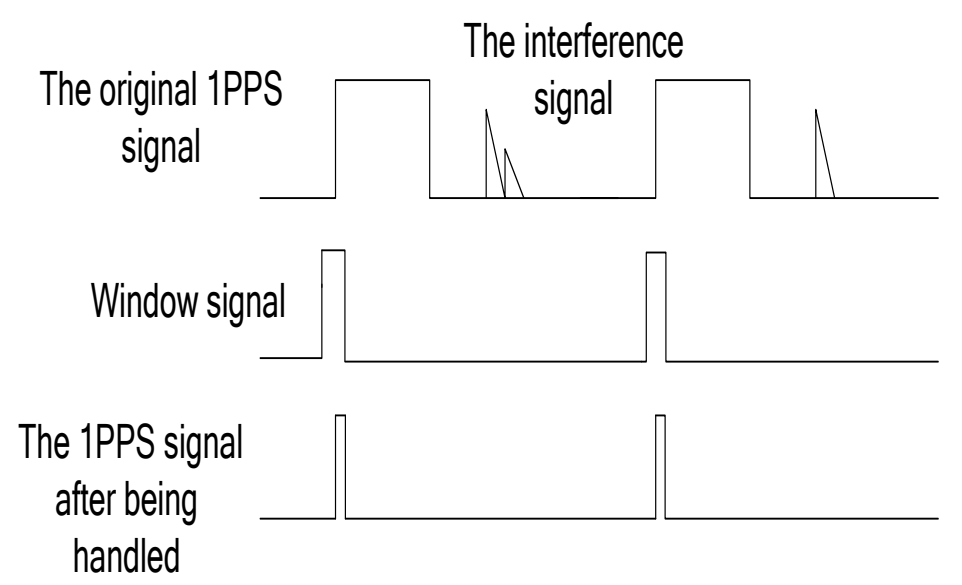

Fig.6 Waveform figure eminating theinterference pulse of 1PPS

\section{Conclusion}

This paper introduces the design of a dynamic online monitoring device based on the precise time service technology beidou satellite and introduces the time service principle of beidou satellite.The devices include the monitoring sub station installed on power transmission line, the monitoring master station installed on the tower and the PC monitor installed in the control center.The method to solve the question that strong electromagnetic interference effects on the beidou satellite time service is put forward.The device can be used to on-line monitor and storage environmental temperature, humidity, wind direction, wind speed, sunshine intensity, conductor temperature and current. The data is wirelessly transmitted to the background expert software,the thermal stability limit of transmission lines is adjusted to maximize the load capacity of transmission lines.

\section{Acknowledgements}

This work was financially supported by financial of National Science Foundation of China (Grant Nos. 51177088).

\section{References}

[1] Qian Zhi-yin,The study of feasibility of the real time dynamic capacity of transmission lines.East-china power,2005.

[2] Guo Hao-kun,The study of Dynamic on-line monitoring device of transmission line.Journal of electric power.2012.

[3] Xu Qing-song,Monitoring of conductor temperature to realize capacity increase technology of transmission lines.Grid technology.2006.

[4] Zhang Bing,The study of Transmission line capacity dynamic monitoring technology.North china electric power university press,2006.

[5] Xiao Li:The study and implementation of real time monitoring and the dynamic capacity of transmission lines.North china electric power university press,2006. 